\title{
Clostridium septicum bacteremia resulting from traumatic myonecrosis: A Case report
}

\section{Melanie Hyte* PharmD, Deep Patel PharmD, Krishna Venkata MD, Carlos Reyes-Sacin MD, AAHISVS FACP FIDSA}

Baptist Medical Center South, Montgomery, Alabama, USA

*Corresponding Author: Melanie Hyte, PharmD, Baptist Medical Centre South, Montgomery, Alabama, USA

Received date: 21 July 2021; Accepted date: 28 July 2021; Published date: 31 July 2021

Citation: Hyte M, Patel D, Venkata K, Reyes-Sacin C, AAHISVS (2021) Clostridium septicum bacteremia resulting from traumatic myonecrosis: A Case report. J Med Case Rep Case Series 2(8): https://doi.org/10.38207/jmcrcs20210101

Copyright: @ 2021 Melanie Hyte. This is an open-access article distributed under the terms of the Creative Commons Attribution License, which permits unrestricted use, distribution, and reproduction in any medium, provided the original author and source are credited.

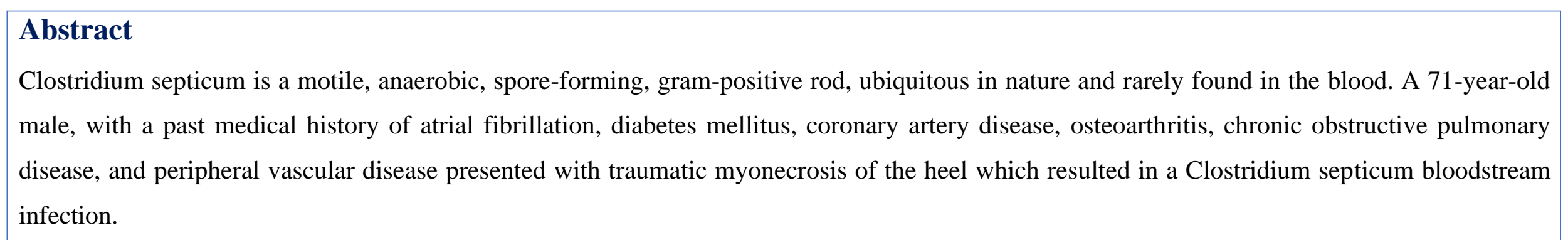

\section{Introduction}

Clostridial myonecrosis (also known as gas gangrene) is a severe infection involving the musculoskeletal tissue originating from a traumatic injury or occurring spontaneously from blood seeding. Clostridium perfringens is responsible for 80 percent of traumatic gas gangrene. The remaining 20 percent are caused by other Clostridium species, one of which is septicum [1]. Clostridium septicum (C. septicum) is responsible for most cases of spontaneous myonecrosis. Clostridium septicum is a gram-positive, obligate anaerobic bacterium that is found in anoxic environments like the human intestinal tract [2]. Bacteremia occurs in approximately 15 percent of those patients with C. septicum. Key treatment considerations for C. septicum infections include the use of broad-spectrum antibiotics with proper debridement.

\section{Case Report}

A 71-year-old Caucasian male with a past medical history of atrial fibrillation, type 2 diabetes mellitus (A1c of $7.4 \%$ ), diabetes-related neuropathy, coronary artery disease, osteoarthritis, chronic obstructive pulmonary disease, and peripheral vascular disease was transferred from an outside facility where he presented with syncope and right heel pain. Upon presentation to the outside facility, the patient had leukocytosis with a white blood cell (WBC) count of $20.9 \times 10^{3}$, hypotension, and a fever of $103 \mathrm{~F}$. Before admission, there was X-ray imaging of gas pockets suspicious for necrotizing fasciitis in the patient's right heel. The patient was transferred to receive a higher level of care. Upon arrival to the facility, the patient had blood and wound cultures obtained and then was started on broad-spectrum antibiotics [intravenous (IV) vancomycin 1,750 mg every 18 hours and IV cefepime 2 gm every 8 hours] and normal saline boluses on admission for possible sepsis. A computed tomography (CT) scan of the lower extremity was performed to evaluate the right heel for
A literature search was performed using PubMed and Google Scholar for similar cases of patients with C. septicum infections. A case series was found with patients with C. septicum bacteremia and risks of colon cancer. [3] C. septicum are frequently the culprit for gastrointestinal infections and peritoneal infections. [4] Clostridium spp. infections usually manifest as gas pockets which mandate anaerobic coverage until culture and sensitivity results can be obtained. The patients with $\mathrm{C}$. septicum infections should also be screened for colon cancer. This organism has also been associated with contaminated musculoskeletal allografts in 14 case reports [6]. The presence of this organism should be considered pathogenic, and treatment should be implemented.

necrotizing fasciitis, which revealed gas gangrene both inferior and posterior to right calcaneus, along with osteomyelitis of the posterior aspect of the right calcaneus. Clindamycin $600 \mathrm{mg}$ IV every 8 hours was added immediately following the CT scan report to cover for necrotizing fasciitis. The infectious diseases (ID) team was consulted to provide recommendations for the management of the gas gangrene. Vascular surgery was also consulted who reported a diabetic foot infection of the right heel with sepsis and necrosis. The patient was taken for debridement of his heel. The tissue and a bone sample were sent for culture and pathology, respectively, to identify the organism and rule out osteomyelitis. Anaerobic cultures were finalized 72 hours post-admission showing $\mathrm{C}$. septicum to be the offending agent. The blood cultures collected at the time of admission resulted in $\mathrm{C}$. septicum growth 8 days after admission. Pathology revealed partially devitalized bone with intertrabecular acute inflammation and necrosis, consistent with acute osteomyelitis. 
Vancomycin and cefepime were discontinued and penicillin G $10,000,000$ units IV every 12 hours was added to the clindamycin therapy. The patient's WBC count trended downwards with a resolution of leukocytosis $\left(8 \times 10^{3}\right) 48$ hours after therapy initiation and the patient remained afebrile during the hospitalization. Two additional debridements were required at nine- and eighteen-days post-admission. The wound culture from the first debridement showed no growth. No repeat blood cultures were ordered. A wound vac was placed on the heel to promote healing. The patient had a

\section{Discussion}

Typically, C. septicum infections originate in the gastrointestinal tract (GI). The report and pathology from the colonoscopy proved that the infection was not linked to a GI issue. Other species of Clostridium are a common causative organism for necrotizing fasciitis, but historically C. septicum has not been a part of this group. The patient's A1c of $7.4 \%$ does not indicate that his diabetes is uncontrolled. Despite appropriate antibiotic treatment, multiple

\section{Conflicts of Interest: None declared}

\section{References}

1. Stevens DL, Bryant A. Clostridial myonecrosis. In: UpToDate. Topic Updated June 25, 2020. Wolters Kluwer. UpToDate, 2021 , Waltham, MA. (Accessed on July 15, 2021).

2. Nanjappa S, Shah S, Pabbathi S (2015) Clostridium septicum Gas Gangrene in Colon Cancer: Importance of Early Diagnosis. Case Reports in Infectious Diseases. 2015: 694247, 3 pages.

3. Description of Clostridium species [Internet]. California: Hardy Diagnostics; c1996-2016 [cited 2021 Apr 21]. colonoscopy two weeks into the admission which showed fragments of tubular adenoma but no neoplasm. The patient was discharged home with a PICC line for administration of penicillin G continuous infusion of 20,000,000 units over 24 hours for a total of 8 weeks. The patient was also prescribed clindamycin $600 \mathrm{mg}$ every 8 hours, by mouth for the same duration. The patient's labs were monitored by the home infusion company and sent to the ID team for follow-up in an outpatient clinic.

debridements were needed for adequate source control; supporting the notion that $\mathrm{C}$. septicum has the potential to be destructive to tissues, even when there are few co-morbidities. This is the first reported case to our knowledge of a patient with bacteremia of C. septicum from a heel wound. Clinicians should exercise timely and aggressive treatment when faced with a Clostridium septicum infection.

4. Bacteremia [Internet]. New Jersey: Merck Manual; 2021 [updated 2020 Jul; cited 2021 Apr 6].

5. Koransky JR, Stargel MD, Dowell VR Jr (1979) Clostridium septicum bacteremia. Its clinical significance. Am J Med. 66(1): 63-6.

6. Kainer MA, Linden JV, Whaley DN, Holmes HT, Jarvis WR, et al. (2004) Clostridium infections associated with musculoskeletal-tissue allografts. N Engl J Med. 50(25): 2564-71. 